\title{
Sequential Changes in Activity of Hip Abductor Muscles after Side-lying Hip Abduction Exercise with Different Directions using Muscle Functional Magnetic Resonance Imaging
}

\author{
Sho Mitomo ${ }^{1,2, *}$, Hideyuki Usa ${ }^{3}$, Masafumi Hata ${ }^{4}$, Daisuke Ogawa ${ }^{5}$, Atsushi Senoo ${ }^{6}$, Hitoshi Takei ${ }^{3}$ \\ ${ }^{1}$ Department of Rehabilitation, Kawakita General Hospital, Suginami-ku, Tokyo, Japan \\ 2Doctoral Course, Department of Physical Therapy, Graduate School of Human Health Sciences, Tokyo Metropolitan University, Arakawa-ku, Tokyo, \\ Japan \\ ${ }^{3}$ Department of Physical Therapy, Graduate School of Human Health Sciences, Tokyo Metropolitan University, Arakawa-ku, Tokyo, Japan \\ ${ }^{4}$ Department of Rehabilitation, Kouseiren Takaoka Hospital, Takaoka-city, Toyama, Japan \\ ${ }^{5}$ Department of Physical Therapy, Faculty of Health Sciences, Mejiro University, Saitama-city, Saitama, Japan \\ ${ }^{6}$ Department of Radiological Sciences, Graduate School of Human Health Sciences, Tokyo Metropolitan University, Arakawa-ku, Tokyo, Japan
}

${ }^{*}$ Corresponding author: Sho Mitomo, Department of Rehabilitation, Kawakita General Hospital, 1-7-3, Asagayakita, Suginami-ku, Tokyo 166-0001, Japan; Tel: +81 3-3339-2553; Fax: +81 3-3339-2121; Email: foreward_numb@yahoo.co.jp

Received: May 20, 2019; Accepted: June 05, 2019; Published: June 12, 2019

\begin{abstract}
Study Design: Controlled laboratory cross-sectional study.

Background: Hip abductor muscle weakness is associated with various lower extremity injuries. Side-lying hip abduction exercises to strengthen the hip abductor muscles is frequently used in rehabilitation and injury prevention programs without scientific evidence regarding their ability to activate the targeted muscles. In addition, previous studies have not quantified the activity of hip abductor muscles during side-lying hip abduction exercises in different directions.
\end{abstract}

Objectives: To measure the T2 values of hip abductor muscles during side-lying hip abduction exercises in different directions using magnetic resonance imaging and to clarify variations in the activity of each segment of the gluteus medius, upper fiber of the gluteus maximus, gluteus minimus, and tensor fasciae latae.

Methods: The T2 values measured using magnetic resonance imaging were used to quantify the activity level of the hip abductor muscles in 10 healthy young males during side-lying hip abduction with different directions (neutral hip, internal rotation and flexion, external rotation, and extension). The two-way repeated measures analysis of variance analysis was used to determine differences between the groups over time.

Results: The T2 values of all muscles, excluding the upper fiber of the gluteus maximus, significantly increased after exercise with all motor tasks over time. The anterior segment of the gluteus medius was significantly increased with side-lying abduction with internal rotation and flexion compared to that with side-lying abduction with external rotation and extension. In contrast, the posterior segments of the gluteus medius and upper fiber of the gluteus maximus were significantly increased with side-lying abduction with external rotation and extension compared to that during other tasks.

Conclusions: The results suggest that side-lying hip abduction exercise with different directions influences the difference in muscle activity between hip abductor muscles and reflects differences in the function of the hip abductor muscles.

Keywords: Hip Abductor Muscles, Transverse Relaxation Times, Muscle Activity, Side-Lying Hip Abduction, Magnetic Resonance Imaging

\section{Introduction}

The hip abductor muscles play an important role in maintaining normal movement patterns of the pelvis and lower extremities. They are considered one of the primary stabilizers in the pelvic region [1]. These muscles build a powerful triangular ensemble spanning between the anterior superior iliac spine, the posterior superior iliac spine, and the greater trochanter region of the femur [2]. Hip abductor muscle weakness has been associated with several lower extremity injuries, including patellofemoral pain syndrome [3-6], iliotibial band friction syndrome [7], anterior cruciate ligament sprains [8-10], and chronic ankle instability [11]. Weakness of the gluteus medius and maximus may contribute to lower extremity injury by influencing joint-loading patterns and lower extremity control $[4,12,13]$. As the hip abductor muscles resist possible injurious motions, such as dynamic knee valgus resulting from excessive hip adduction and internal rotation, 
improvement of hip abductor muscle strength and activation may be a critical aspect of rehabilitation and injury prevention programs [14].

Although hip abductor muscles at the anatomic site have not been defined completely, several studies have reported that the varying anatomic structures of the hip abductor muscle fibers translate to differences in function [7,15-20]. Many reports about rehabilitation programs for the hip abductor muscles based on the presence of the functional subdivisions are available.

Electromyography (EMG) is one of the most reliable ways to evaluate skeletal muscle activity. Recent studies have sought to determine which exercises are the best to activate the hip abductor muscles, consisting of the Gluteus Medius (GMED), Gluteus Minimus (GMIN), Tensor Fasciae Latae (TFL), and Gluteus Maximus [21-28]. Distefano et al. reported that gluteus medius activity is significantly greater during side-lying hip abduction compared with that during other exercises such as clam exercises, lunges, and hop exercises [29]. Side-lying abduction exercise is frequently used clinically in rehabilitation sessions because it can be performed early in a rehabilitation program to generate proper neuromuscular control and strength since it is less demanding than an open kinematic chain exercise [29]. While EMG is a valuable instrument, significant limitations also exist in its use as an indicator of muscle function. EMG recorded from surface electrodes may be contaminated by crosstalk from the surrounding muscles [30, 31]. EMG signal detected using fine-wire electrodes is specific to the target/sampled muscle, and the normalized intramuscular signal is representative of the entire muscles [32]. However, this method for measuring muscle activation is invasive.

Muscle Functional Magnetic Resonance Imaging (mfMRI) can quantify all muscle activities within the imaging range by exploiting the process by which exercise induces signal changes that result primarily from increases in the transverse relaxation time (T2) of water in the tissues. T2 changes measured with mfMRI have high validity[33, 34] and reliability.[35, 36] Therefore, because the prolongation in T2 relaxation time could easily be used as a noninvasive, quantitative measurement for muscle activity of the deep muscles, this technique is an excellent tool for assessing the extent of muscle activation following the performance of a task [37].

Kumagai et al [38] investigated the activity of the GMIN and that of the deep and superficial layers of the GMED. They demonstrated using the T2 values that the activity levels of these differing portions of the abductor muscles are influenced by the degree of hip abduction angle during isometric hip abduction exercise. Recently, Mitomo et al. reported that side-lying hip abduction exercise immediately increases the rate change in the T2 value of the TFL, GMIN, and the anterior and middle segments of the GMED but delays the activation of the upper fiber of the Gluteus Maximus (UGM) and posterior segment of the GMED [39]. These reports demonstrated that the activity levels of the muscles with hip abduction action were not homogeneous, and a functional difference existed between the hip abductor muscles. However, the exact role of functional subdivisions of hip abductor muscle remains poorly understood. Isotonic exercise strengthens the muscle involved in joint movement and can be effective in strengthening muscles at various angles, which are often used in clinical practice. Although many studies on changes in hip abductor activity during isometric side-lying hip abduction exercise have been reported, there has been no study on the muscle activity of the hip abductor during isotonic side-lying hip abduction exercise in different directions using the $\mathrm{T} 2$ values. Therefore, this study aimed to measure the $\mathrm{T} 2$ values of the hip abductor muscles after side-lying hip abduction exercise with different directions over time and to clarify variations in the activity of the hip abductor muscles.

\section{Methods \& Materials}

\section{Subjects}

Ten young healthy males with a mean age of 26.1 (range 24-32) years, a mean (SD) height of $1.72(0.04) \mathrm{m}$, and a mean weight of 63.3 (8.1) kg participated in the study. Subjects were excluded if they reported any musculoskeletal disorders of the trunk or lower extremities or any neurological conditions. Written informed consent was obtained from all subjects after the aims of the study and its protocol had been explained to them in detail. The study protocol was reviewed and approved by the Ethics Committee of Tokyo Metropolitan University.

\section{Acquisition of Magnetic Resonance Images}

A 3.0-T MRI system (Achieva 3.0T; Philips, Tokyo, Japan) was used for all patients. T2 mapping was performed in addition to routine T2-weighted imaging. T2 measurement with single-slice acquisition was performed on the upper part of the acetabulum using a turbo spin echo sequence. The turbo spin echo scanning parameters were as follows: 6 echo times of 13-78 ms; repetition time, $4200 \mathrm{~ms}$; field of view, $350 \times 350 \mathrm{~mm}$; matrix size, $269 \times 269$; slice thickness, $5.0 \mathrm{~mm}$; and number of slices, 7 .

\section{T2 Measurement}

The images were processed using a DICOM viewer (OsiriX Lite, Pixmeo Sàrl, Geneva, Switzerland) to determine the relaxation times. A water capsule was placed along each segment of the GMED to distinguish each type of fiber. The T2 values were measured for the TFL; GMIN; anterior, middle, and posterior segments of the GMED; and UGM. Four regions of interest (ROIs), each located in the TFL and GMIN and anterior, middle, and posterior segments of the GMED, were determined to investigate the changes in signal intensity. For the UGM, five ROIs were identified to assess the changes in signal intensity. The ROIs were manually selected using a computer mouse, after which the mean of the T2 values between the pixels of the ROI was automatically calculated using the OsiriX Lite software (Figure 1). Care was taken to exclude subcutaneous and intramuscular fat, aponeuroses, and vessels from the selected regions. The T2 values for each muscle were taken as the mean value of T2 for the selected ROIs, and the workload for each muscle was expressed as a $\mathrm{T} 2$ value in milliseconds.

\section{Exercise Protocol}

The subjects performed 5 sets of 40 repetitions of a hip abduction exercise at $30 \%$ maximum voluntary contraction with the right leg 
during each task. Isometric maximum voluntary contraction of each task was measured, from which we calculated the $30 \%$ maximum voluntary contraction of each task. The pelvis was fixed with a belt to avoid compensatory movements as much as possible. MRI scans were performed before exercise, at intervals during exercise, and at the end of the exercise. The exercise was performed inside the magnet bore of the MRI scanner, and the subjects were then immediately moved into the magnet for imaging.

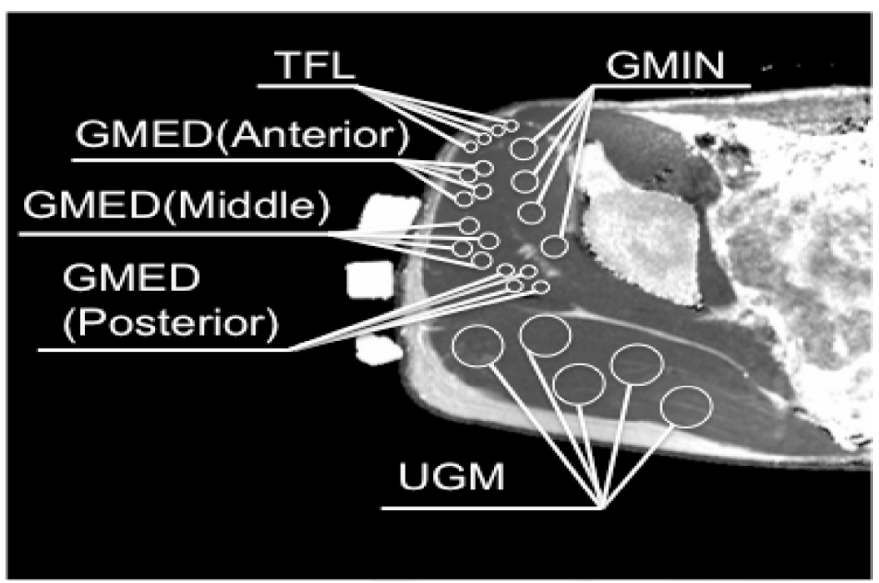

Figure 1. T2 measurement of hip abductor muscles on T2 calculated map. GMED, gluteus medius; GMIN, gluteus minimus; TFL, tensor fascia latae; UGM, upper fiber of the gluteus maximus.

\section{Task 1: Side-lying hip abduction with neutral hip (Figure 2A)}

Subjects lay on their sides with the upper trunk and pelvis aligned in a straight line on the treatment table. The bottom side of the hip joint was flexed at $45^{\circ}$, and the knee joints were flexed at $90^{\circ}$ for stabilization. A plastic target bar was placed at $20^{\circ}$ of the hip abduction range of motion. The movement direction was indicated using a plastic plate, which was placed vertical to the floor. The subjects abducted the hip joint along the plate. In each subject, the hip was abducted $20^{\circ}$ over $1 \mathrm{~s}$ and then returned to its initial position over $1 \mathrm{~s}$. No rest periods were allowed during exercise. The subjects were cued to point their toes forward by abduction from the hip as much as they could without rotating their pelvis forward or backward.

Task 2: Side-lying hip abduction with internal rotation and flexion (Figure 2B)

Subjects performed this task in the same manner as in side-lying abduction with neutral hip, excluding the hip internal rotation and flexion. The movement direction was indicated using a plastic plate. The plastic plate was placed to tilt $30^{\circ}$ forward from the vertical plane on the floor. Subjects performed side-lying hip abduction exercise up to abduction $20^{\circ}$ in a forward direction of $30^{\circ}$ with internal rotation along the plate to avoid pressing the plate as much as possible. The subjects were cued to point their toes toward the floor by rotating from the hip as much as they could, without rotating their pelvis forward or backward.

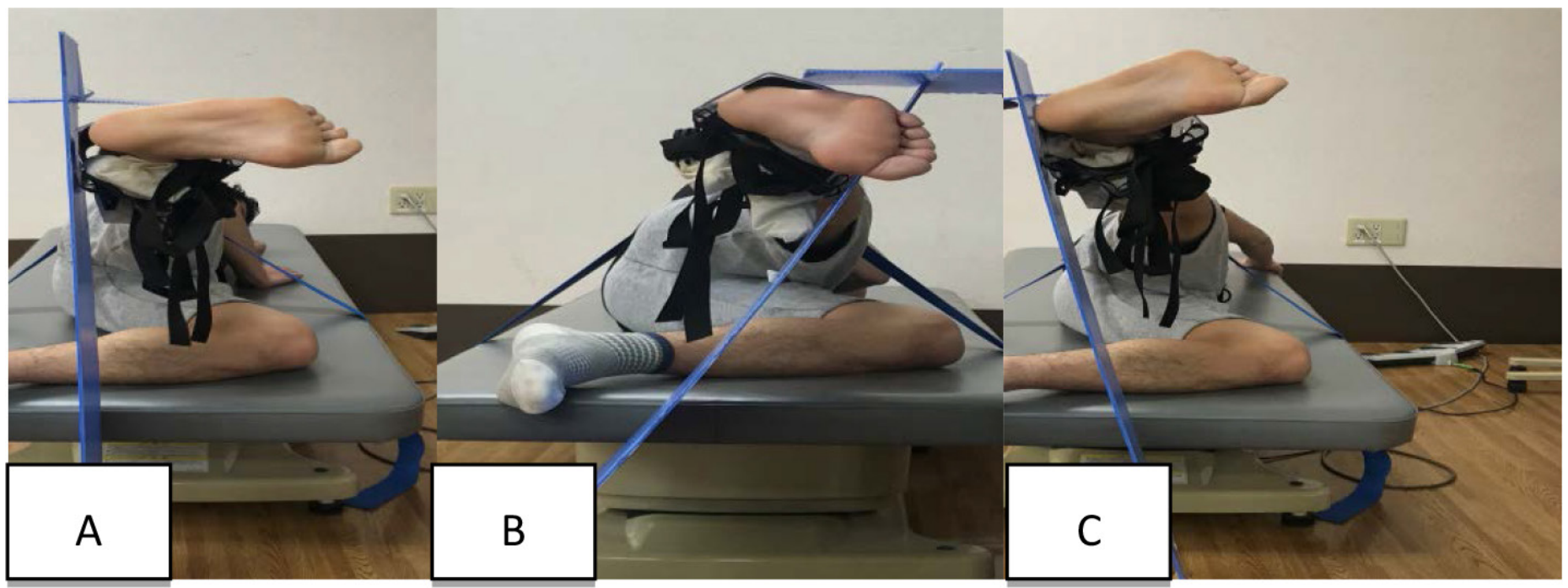

Figure 2. Subject performing the exercise task.

A: Side-lying hip abduction with neutral hip.

B: Side-lying hip abduction with internal rotation and flexion.

C: Side-lying hip abduction with external rotation and extension.

Task 3: Side-lying hip abduction with external rotation and extension (Figure 2C)

Subjects performed this task in the same manner as in side-lying abduction with neutral hip, excluding the hip external rotation and flexion. The movement direction was indicated using a plastic plate.
The plastic plate was placed to tilt $10^{\circ}$ backward from the vertical plane on the floor. The subjects performed side-lying hip abduction exercise up to $20^{\circ}$ abduction in a backward direction of $10^{\circ}$ with external rotation along the plate to avoid pressing the plate as much as possible. The subjects were cued to point their toes toward the ceiling 
Mitomo S (2019) Sequential Changes in Activity of Hip Abductor Muscles after Side-Lying Hip Abduction Exercise with Different Directions Using Muscle Functional Magnetic Resonance Imaging

by rotating from the hip as much as they could, without rotating their pelvis forward or backward.

\section{Statistical Analysis}

For each muscle, we used the two-way repeated measures analysis of variance (ANOVA) with the main effect being task (task 1, task 2, and task 3 ) and exercise set (pre-exercise, 1, 2, 3, 4, and 5 sets). All data were analyzed at an alpha level of .05. Significant differences from the ANOVA were further examined using Bonferroni post hoc analysis, with the alpha level corrected for multiple comparisons of less than .05. All statistical analyses were performed using SPSS version 22
(IBM Corporation, Armonk, NY), and outcome data were presented as mean (range) or mean (SD).

\section{Results}

No significant exercise task-by-exercise set interactions were found for the T2 values of the TFL, GMIN, and middle segment of the GMED (Table 1). However, there were main effects in these muscles for the exercise set (Table 1). Bonferroni post hoc analysis comparing the exercise set revealed that the T2 values of these muscles had a significant increase after exercise (Table 2, 3, 5).

Table 1. Two-way repeated measures analysis of variance for comparisons between the exercise task and exercise set.

\begin{tabular}{|c|c|c|c|c|c|c|c|c|c|c|c|c|}
\hline & \multicolumn{2}{|c|}{ TFL } & \multicolumn{2}{|c|}{ GMIN } & \multicolumn{2}{|c|}{ GMED (anterior) } & \multicolumn{2}{|c|}{ GMED (middle) } & \multicolumn{2}{|c|}{ GMED (posterior) } & \multicolumn{2}{|c|}{ UGM } \\
\hline & F values & Pvalues & F values & Pvalues & F values & Pvalues & F values & P values & F values & P values & F values & Pvalues \\
\hline Task & 0.731 & 0.495 & 1.127 & 0.346 & 3.624 & 0.048 & 2.386 & 0.120 & 15.074 & 0.000 & 7.650 & 0.000 \\
\hline Exercise set & 27.678 & 0.000 & 118.849 & 0.000 & 92.689 & 0.000 & 44.310 & 0.000 & 38.312 & 0.000 & 9.465 & .0 .002 \\
\hline Task $\times$ exercise set & 0.671 & 0.749 & 0.606 & 0.805 & 2.031 & 0.039 & 0.735 & 0.690 & 4.228 & 0.000 & 2.972 & 0.003 \\
\hline
\end{tabular}

TFL, tensor fasciae latae; GMIN, gluteus minimus; GMED, gluteus medius; UGM, upper fiber of the gluteus maximus

Table 2. Comparison of the T2 values of the TFL (mean (SD), ms) according to exercise task and exercise set

\begin{tabular}{|c|c|c|c|c|c|c|c|}
\hline & Pre-exercise & 1 set & 2 sets & 3 sets & 4 sets & 5 sets & $\begin{array}{c}\text { Multiple comparisons } \\
\text { (exercise set) }\end{array}$ \\
\hline Task 1 & $31.8(0.8)$ & $35.3(2.4)$ & $36.7(3.0)$ & $38.8(3.1)$ & $38.9(3.0)$ & $39.1(2.9)$ & \multirow{3}{*}{$\begin{array}{c}\text { Pre-exercise }<1,2,3,4,5 \text { sets } \\
1 \text { set }<3,4,5 \text { sets }{ }^{\dagger} \\
2 \text { sets }<3,4,5 \text { sets }^{\ddagger}\end{array}$} \\
\hline Task 2 & $32.1(0.9)$ & $36.2(3.8)$ & $37.5(4.7)$ & $39.0(5.5)$ & $38.9(5.1)$ & $38.6(4.7)$ & \\
\hline Task 3 & $32.0(1.3)$ & $36.8(3.5)$ & $38.3(4.4)$ & $39.2(5.1)$ & $39.6(4.6)$ & $39.6(4.0)$ & \\
\hline
\end{tabular}

Multiple comparisons (exercise set): the result of comparing the $\mathrm{T} 2$ values of each exercise set during all exercise tasks using Bonferroni post hoc analysis.

${ }^{*} \mathrm{p}=0.017,0.019,0.008,0.004,0.003$, respectively; ${ }^{\dagger} \mathrm{p}=0.013,0.006,0.001$, respectively; ${ }^{\dagger} \mathrm{p}=0.001,0.006,0.002$, respectively

Table 3. Comparison of the T2 values of the GMIN (mean (SD), ms) according to exercise task and exercise set

\begin{tabular}{|c|c|c|c|c|c|c|c|}
\hline & Pre-exercise & 1 set & 2 sets & 3 sets & 4 sets & 5 sets & Multiple comparisons (exercise set) \\
\hline Task 1 & $35.2(1.6)$ & $40.2(1.6)$ & $41.4(1.5)$ & $42.7(2.2)$ & $41.7(1.6)$ & $40.6(1.3)$ & $\begin{array}{c}\text { Pre-exercise }<1,2,3,4,5 \text { sets* } \\
1 \text { set<2,3 sets } \\
4 \text { sets }>5 \text { sets }{ }^{\dagger}\end{array}$ \\
\hline Task 2 & $35.5(1.6)$ & $41.0(2.0)$ & $42.4(2.1)$ & $42.7(2.0)$ & $42.5(2.4)$ & $41.1(2.4)$ \\
\hline Task 3 & $35.3(1.3)$ & $41.1(1.7)$ & $41.5(2.6)$ & $41.9(1.7)$ & $41.8(1.2)$ & $41.1(1.4)$ \\
\hline
\end{tabular}

Multiple comparisons (exercise set): the result of comparing the T2 values of each exercise set during all exercise tasks using Bonferroni post hoc analysis

${ }^{*} \mathrm{p}=0.000$, for all sets; ${ }^{\dagger} \mathrm{p}=0.009,0.001$, respectively; ${ }^{\star} \mathrm{p}=0.001$

Table 4. Comparison of the $\mathrm{T} 2$ values of the anterior segment of the GMED (mean (SD), ms) according to exercise task and exercise set

\begin{tabular}{|c|c|c|c|c|c|c|}
\hline & Pre-exercise & 1 set & 2 sets & 3 sets & 4 sets & Multiple comparison \\
(exercise set)
\end{tabular}

Multiple comparisons (exercise set): the result of comparing the T2 values of each exercise set at each exercise task using Bonferroni post hoc analysis. Multiple comparisons (exercise task): the result of comparing the T2 values of each exercise task at each exercise set using Bonferroni post hoc analysis ${ }^{*} \mathrm{p}=0.000$, for all sets; ${ }^{\dagger} \mathrm{p}=0.000$, for all sets; ${ }^{\dagger} \mathrm{p}=0.000 ;{ }^{\S} \mathrm{p}=0.001,0.003,0.000,0.000,0.000$, respectively; ${ }^{*} \mathrm{p}=0.046 ;{ }^{\dagger} \mathrm{p}=0.041$ 
Mitomo S (2019) Sequential Changes in Activity of Hip Abductor Muscles after Side-Lying Hip Abduction Exercise with Different Directions Using Muscle Functional Magnetic Resonance Imaging

Table 5. Comparison of the T2 values of the middle segment of the GMED (mean (SD), ms) according to exercise task and exercise set.

\begin{tabular}{|c|c|c|c|c|c|c|}
\hline & Pre-exercise & 1 set & 2 sets & 3 sets & 4 sets & Multiple comparisons (exercise \\
set) & & $39.0(2.0)$ & $40.0(2.1)$ & $39.6(2.0)$ & $39.7(2.0)$ \\
\hline Task 1 & $35.0(1.4)$ & $38.6(2.7)$ & $40.1(3.1)$ & $40.7(4.0)$ & $40.8(3.3)$ & $40.3(3.5)$ \\
\hline Task 2 & $35.8(1.7)$ & $39.0(1.7)$ & $38.6(2.9)$ & $39.0(2.7)$ & $38.9(2.2)$ & $39.2(2.1)$ \\
\hline Task 3 & $34.8(1.7)$ & $38.7(3.3)$ & & \\
\hline
\end{tabular}

Multiple comparisons (exercise set): the result of comparing the T2 values of each exercise set during all exercise tasks using Bonferroni post hoc analysis $* \mathrm{p}=0.000$, for all sets

In contrast, a significant exercise task-by-exercise set interaction was found for the T2 values of the anterior and posterior segments of the GMED and UGM (Table 1).

For the anterior segment of the GMED, Bonferroni post hoc analysis comparing each exercise task revealed that the T2 values at task 2 was significantly increased compared with task 3 at 4 and 5 sets (Table 4). In addition, the T2 values of all tasks were significantly increased after exercise.

For the posterior segment of the GMED, Bonferroni post hoc analysis comparing each task revealed that task 3 was significantly higher than task 1 after all exercise sets, and was significantly higher than task 2 after 2, 3, 4, and 5 sets (Table 6). In addition, the T2 values of all tasks were significantly increased after exercise (Table 6).

For the UGM, Bonferroni post hoc analysis comparing each task revealed that task 3 was significantly higher than task 1 after 5 sets and was significantly higher than task 2 after 4 sets and 5 sets (Table 7). In addition, only the $\mathrm{T} 2$ values of task 3 were significantly increased after exercise (Table 7).

Table 6. Comparison of the T2 values of the posterior segment of the GMED (mean (SD), ms) according to exercise task and exercise set.

\begin{tabular}{|c|c|c|c|c|c|c|}
\hline & Pre-exercise & 1 set & 2 sets & 3 sets & Multiple comparisons \\
(exercise set)
\end{tabular}

Multiple comparisons (exercise set): the result of comparing the T2 values of each exercise set at each exercise task using Bonferroni post hoc analysis

Multiple comparisons (exercise task): the result of comparing the T2 values of each exercise task at each exercise set using Bonferroni post hoc analysis.

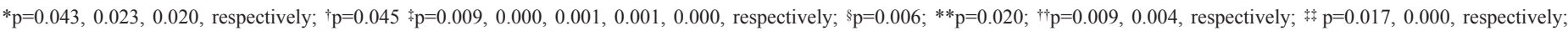
$\S \S \mathrm{p}=0.009,0.001$, respectively; $* * * \mathrm{p}=0.002,0.002$, respectively

Table 7. Comparison of the T2 values of the UGM (mean (SD), ms) according to exercise task and exercise set.

\begin{tabular}{|c|c|c|c|c|c|c|c|}
\hline & Pre-exercise & $1 \mathrm{set}$ & 2 sets & 3 sets & 4 sets & 5 sets & Multiple comparisons (exercise set) \\
\hline Task 1 & $37.4(1.4)$ & $38.1(1.2)$ & $38.4(1.4)$ & $38.5(1.2)$ & $38.6(1.2)$ & $38.1(1.1)$ & n.s. \\
\hline Task 2 & $38.0(2.0)$ & $38.4(1.4)$ & $38.6(1.9)$ & $38.6(1.7)$ & $38.8(1.5)$ & $38.5(2.0)$ & n.s. \\
\hline Task 3 & $37.2(1.6)$ & $38.8(1.4)$ & $39.3(1.4)$ & $40.1(1.4)$ & $40.6(1.5)$ & $40.3(1.0)$ & $\begin{array}{c}\text { Pre-exercise }<2,3,4,5 \text { sets }^{\star} \\
1 \text { set }<3,4,5 \text { sets }^{\dagger} \\
2 \text { sets }<5 \text { sets }^{\ddagger}\end{array}$ \\
\hline $\begin{array}{c}\text { Multiple } \\
\text { comparisons } \\
\text { (exercise task) }\end{array}$ & n.s. & n.s. & n.s. & n.s. & Task $3>$ Task $2^{\S}$ & Task $3>$ Tasks $1,2^{* *}$ & \\
\hline
\end{tabular}

Multiple comparisons (exercise set): the result of comparing the T2 values of each exercise set at each exercise task using Bonferroni post hoc analysis

Multiple comparisons (exercise task): the result of comparing the T2 values of each exercise task at each exercise set using Bonferroni post hoc analysis.

${ }^{*} \mathrm{p}=0.000,0.001,0.001,0.000$, respectively; ${ }^{\mathrm{p}} \mathrm{p}=0.040,0.016,0.000$, respectively; ${ }^{\star} \mathrm{p}=0.045 ;{ }^{\S} \mathrm{p}=0.043 ;{ }^{* *} \mathrm{p}=0.013,0.025$, respectively. 


\section{Discussion}

In this study, the $\mathrm{T} 2$ values of the hip abductors increased with an increasing load of exercise. The results of this study demonstrated that the T2 values could be used to assess muscle activity. Many factors could contribute to the changes in $\mathrm{T} 2$, including increases in intracellular and extracellular water content, accumulation of diamagnetic ions (e.g., lactate, phosphate, and sodium), and a decrease in $\mathrm{pH}[40,41]$. $\mathrm{T} 2$ shift measures provide a powerful technique to assess muscle function during specific exercise/rehabilitation protocols [37]. The muscle activation data evaluated using the $\mathrm{T} 2$ values associated with exercise in the present study were consistent with those in a previous study caused by the factors described above. Our results demonstrated that the hip abductor muscles were activated differently between the side-lying hip abduction exercise variations examined.

The T2 values of the TFL in all tasks were increased over time, and no significant difference was found between each task. The TFL is located in the superficial layer [15], and its primary role is abduction of the hip joint as well as flexion and internal rotation [17, 42]. Gottschalk et al.[17] proposed in their muscle modeling studies that the main function of the TFL is hip abduction. Sidorkewicz et al.[43] found that the activity of the TFL does not vary significantly during hip abduction exercise with neutral hip and internal and external rotations, which was in agreement with our findings.

The T2 values of the GMIN in all tasks were increased over time, and no significant difference was found between each task. The GMIN is located in the deepest layer, and its muscle belly adheres directly to the superior joint capsule [44], which enables this muscle to augment and protect joint stability $[44,45]$. Based on anatomic and EMG studies, the primary function of the entire GMIN is to stabilize the head of the femur in the acetabulum [17]. Therefore, the GMIN was activated in all tasks to stabilize the head of the femur in the acetabulum during exercise because of its anatomical structure and function.

No significant difference was found between the tasks in the T2 of the middle segments of the GMED; this result is probably attributable to its anatomical structure. Middle fascicles have been reported to be more vertically oriented, which appears to be a better position to abduct the hip [1]. Therefore, it is suggested that the middle segment of the GMED contracts due to the element of hip abduction of the exercise task performed in this study.

The activity of the anterior segments of the GMED was increased in all tasks over time. Additionally, the T2 values of the anterior segment of the GMED were increased in task 2 compared to those in task 3. On the other hand, the activity of the posterior segment of the GMED in task 3 was increased immediately, and the T2 values in task 3 were increased compared to those in other tasks. Our results appear to reflect the concept in which task-dependent activation differences of various segments of the GMED indicate a functional subdivision within the muscle. Cadaveric and anatomical studies suggest that the GMED comprises three structurally unique regions (anterior, middle, and posterior) $[18,46-48]$, the activity of which may be independent of the central nervous system control $[18,19]$. The patterns of orientation and insertion of the anterior and posterior portions of the gluteus medius appear to reflect their probable role in internal and external rotations, respectively, and are in line with the findings of EMG studies $[1,16]$. Semciw et al. [49] studied the activity of each segment of the GMED during hip exercise and demonstrated using fine-wire EMG that muscle activation in the posterior GMED during the clam maneuver is higher than that in other segments of GMED. Hip movement of the clam maneuver is abduction with extension and external rotation, and side-lying hip abduction with extension and external rotation in the current study is similar to the clam maneuver. Therefore, the current study showed that the activity of the anterior and posterior segments of the GMED was in agreement with a previous research. O'Sullivan et al.[50] noted that the presence of these subdivisions may require exposure of the degree of muscle activity for each subdivision during a variety of clinically used strengthening exercises. Therefore, given these results, we suggest performing side-lying hip abduction with extension and external rotations as an effective method to activate the posterior segment of the GMED.

The T2 values of the UGM were increased in task 3 compared to those in other tasks, similar to the posterior segment of the GMED. The UGM is located in the superficial layer [15], and because of its anatomical structure, its primary role is abduction of the hip joint as well as extension and external rotation [42]. Our findings are in agreement with those of Selkowitz et al.[51] who reported that the superior gluteus maximus EMG activity is greater than the incorporated hip abduction and/or external rotation movements. Thus, the results of the T2 values in the UGM showed that side-lying hip abduction with external rotation and extension activated compared with the sidelying hip abduction with neutral hip or internal rotation and flexion.

This study has several limitations. First, real-time muscle activity during exercise could not be evaluated using the T2 values. However, in this study, the T2 values were measured immediately after exercise. Thus, interpretation of the change in the $\mathrm{T} 2$ values is related to all the work performed by the muscle and not just to a single activity. An exercise-induced shift in T2 is detectable after a few as two contractions and increases to a work-rate-dependent plateau within a few minutes [40]. Recovery after exercise takes at least 20 min [52], which should have enabled us to measure exercise-induced shifts in T2 after exercise. Second, this study did not evaluate muscle activity using EMG. Even if there was no significant change in the intensity of the MRI signal, the work of the muscle may possibly be observed on EMG. Third, as the exercise load increases, a synergistic contraction of other hip joint muscles exists during hip abduction exercise, but the $\mathrm{T} 2$ values of other hip joint muscles were not measured. In addition, the exercise task had only 3 conditions, and the variation of other hip abduction motion was not considered. However, this study confirmed the movement of free water inside and outside of muscle cells when the activity level increased in the hip abductor muscles. The results of our study suggest that the variation in changes in activity observed between the hip abductor muscles was attributable to the differences in their anatomic structure and was indicative of intramuscular variation of activity within the hip abductor muscles.

Hip-focused neuromuscular exercise interventions have gained considerable attention for addressing a myriad of lower extremity injuries [53]. Deficits in proximal hip strength or neuromuscular control may lead to lower extremity valgus [9]. Dynamic lower 
Mitomo S (2019) Sequential Changes in Activity of Hip Abductor Muscles after Side-Lying Hip Abduction Exercise with Different Directions Using Muscle Functional Magnetic Resonance Imaging

extremity valgus is operationally defined as a combination of motions and rotations in the lower extremity, including hip adduction and internal rotation, knee abduction, and tibial external rotation [8]. Therefore, the posterolateral hip musculature has hip abduction and external rotation, play a central role in controlling the dynamic alignment of the lower extremity. The current study demonstrated that posterolateral hip musculature, such as the posterior segment of the GMED and UGM, was activated during side-lying hip abduction with extension and external rotation. Thus, this knowledge will allow physical therapists to develop specific and targeted rehabilitation programs for these muscles and clinical condition. However, this suggestion needs validation through further research involving people with lower extremity conditions. Whether activation of the posterior segment of the GMED and UGM could improve lower limb kinematics and athletic performance should also be validated.

\section{Acknowledgment}

We thank the subjects and the other members of the study group for their participation. This study would not have been possible without them.

\section{References}

1. Conneely M, O'Sullivan K (2008) Gluteus maximus and gluteus medius in pelvic and hip stability: isolation or synergistic activation? Physio Ireland 29: 6-10.

2. Anders C, Patenge C, Sander K, Layher F, et al. (2017) Detailed spatial characterization of superficial hip muscle activation during walking: a multielectrode surface EMG investigation of the gluteal region in healthy older adults. PLoS One 1: 1-24.

3. Cichanowski HR, Schmitt JS, Johnson RJ, Niemuth PE (2007) Hip strength in collegiate female athletes with patellofemoral pain. Med Sci Sports Exerc 39: 1227-1232. [ crossref]

4. Ireland ML, Willson JD, Ballantyne BT, Davis IM (2003) Hip strength in females with and without patellofemoral pain. J Orthop Sports Phys Ther 33: 671-676. [ crossref]

5. Robinson RL, Nee RJ (2007) Analysis of hip strength in females seeking physical therapy treatment for unilateral patellofemoral pain syndrome. J Orthop Sports Phys Ther. 37: 232-238.

6. Rowe J, Shafer L, Kelley K, West N, Dunning T, et al. (2007) Hip strength and knee pain in females. N Am J Sports Phys Ther 2: 164-169. [ crossref]

7. Fredericson M, Cookingham CL, Chaudhari AM, Dowdell BC, Oestreicher N, et al. (2000) Hip abductor weakness in distance runners with iliotibial band syndrome. Clin J Sport Med 10: 169-175. [ crossref]

8. Hewett TE, Myer GD, Ford KR (2006) Anterior cruciate ligament injuries in female athletes: Part 1, mechanisms and risk factors. Am J Sports Med 34: 299-311.

9. Hewett TE, Myer GD, Ford KR, Heidt RS Jr, et al. (2005) Biomechanical measures of neuromuscular control and valgus loading of the knee predict anterior cruciate ligament injury risk in female athletes: a prospective study. Am J Sports Med 33: 492-501.

10. Ireland ML (2002) The female ACL: why is it more prone to injury? Orthop Clin North Am 33: 637-651. [ crossref]

11. Friel K, McLean N, Myers C, Caceres M (2006) Ipsilateral hip abductor weakness after inversion ankle sprain. J Athl Train 41: 74-78.

12. Fulkerson JP (2002) Diagnosis and treatment of patients with patellofemoral pain. Am J Sports Med 30: 447-456. [ crossref]

13. Powers CM (2003) The influence of altered lower-extremity kinematics on patellofemoral joint dysfunction: a theoretical perspective. J Orthop Sports Phys Ther 33: 639-646.

14. Distefano LJ, Blackburn JT, Marshall SW, Padua DA (2009) Gluteal muscle activation during common therapeutic exercises. J Orthop Sports Phys Ther 39: $532-540$.

15. Grimaldi A, Richardson C, Hides J, Donnelly W, et al. (2009) The association between degenerative hip joint pathology and size of the gluteus maximus and tensor fascia lata muscles. Man Ther 14: 611-617.

16. Earl JE (2005) Gluteus medius activity during three variations of isometric singleleg stance. J Sport Rehabil 14: 1-11.
17. Gottschalk F, Kourosh S, Leveau B (1989) The functional anatomy of tensor fasciae latae and gluteus medius and minimus. J Anat 166: 179-189. [ crossref]

18. Schmitz RJ, Riemann BL, Thompson T (2002) Gluteus medius activity during isometric closed-chain hip rotation. $J$ Sport Rehabil 11(3): 179-188.

19. Soderberg G, Dostal W (1978) Electromyographic study of three parts of the gluteus medius muscle during functional activities. Phys Ther 58: 691-696.

20. Wilson G, Capen E, Stubbs N (1976) A fine-wire electromyographic investigation of the gluteus medius and minimus muscles. Res Q 47: 824-828.

21. Arokoski JP, Kankaanpää M, Valta T, Juvonen I, et al. (1999) Back and hip extensor muscle function during therapeutic exercises. Arch Phys Med Rehabil 80: 842-850.

22. Ayotte NW, Stetts DM, Keenan G, Greenway EH (2007) Electromyographical analysis of selected lower extremity muscles during 5 unilateral weight-bearing exercises. J Orthop Sports Phys Ther. 37: 48-55.

23. Bolgla LA, Uhl TL (2005) Electromyographic analysis of hip rehabilitation exercises in a group of healthy subjects. J Orthop Sports Phys Ther 35: 487-494.

24. Bolgla LA, Uhl TL (2007) Reliability of electromyographic normalization methods for evaluating the hip musculature. J Electromyogr Kinesiol 17: 102-111.

25. Boudreau SN, Dwyer MK, Mattacola CG, Lattermann C, Uhl TL, et al. (2009) Hip-muscle activation during the lunge, single-leg squat, and step-up-and-over exercises. J Sport Rehabil 18: 91-103. [ crossref]

26. Ekstrom RA, Donatelli RA, Carp KC (2007) Electromyographic analysis of core trunk, hip, and thigh muscles during 9 rehabilitation exercises. J Orthop Sports Phys Ther 37: 754-762.

27. McBeth JM, Earl-Boehm JE, Cobb SC, Huddleston WE (2012) Hip muscle activity during 3 side-lying hip-strengthening exercises in distance runners. $J$ Athl Train 47: $15-23$.

28. Lee JH, Cynn HS, Kwon OY, Yi CH, et al (2014) Different hip rotation influence hip abductor muscles activity during isometric side-lying hip abduction in subjects with gluteus medius weakness. J Electromyogr Kinesiol 24: 318-324.

29. Distefano LJ, Blackburn JT, Marshall SW, Padua DA (2009) Gluteal muscle activation during common therapeutic exercises. J Orthop Sports Phys 39: 532-540.

30. Johnson VL, Halaki M, Ginn KA (2011) The use of surface electrodes to record infraspinatus activity is not valid at low infraspinatus activation levels. $J$ Electromyogr Kinesiol 21: 112-118.

31. Perry J, Easterday CS, Antonelli DJ (1981) Surface versus intramuscular electrodes for electromyography of superficial and deep muscles. Phys Ther 61: 7-15.

32. Bogey RA, Perry J, Bontrager EL, Gronley JK (2000) Comparison of acrosssubject EMG profiles using surface and multiple indwelling wire electrodes during gait. J Elecromyogr Kinesiol. 10: 255-259.

33. Adams GR, Duvoisin MR, Dudley GA (1992) Magnetic resonance imaging and electromyography as indexes of muscle function. J Appl Physiol (1985) 73: 15781583. [ crossref]

34. Dickx N, D’Hooge R, Cagnie B, Deschepper E, et al. (2010) Magnetic resonance imaging and electromyography to measure lumbar back muscle activity. Spine (Phila Pa 1976) 35: 836-842.

35. Elliott JM, O'Leary SP, Cagnie B, Durbridge G, et al. (2010) Craniocervical orientation affects muscle activation when exercising the cervical extensors in healthy subjects. Arch Phys Med Rehabil 91: 1418-1422.

36. Cagnie B, Dickx N, Peeters I, Tuytens J, et al. (2008) The use of functional MRI to evaluate cervical flexor activity during different cervical flexion exercises. $J \mathrm{Appl}$ Physiol 104: 230-235.

37. Cagnie B, Elliott JM, O'Leary S, D'hooge R, Dickx N, et al. (2011) Muscle functional MRI as an imaging tool to evaluate muscle activity. $J$ Orthop Sports Phys Ther 41: 896-903. [ crossref]

38. Kumagai M, Shiba N, Higuchi F, Nishimura H, et al. (1997) Functional evaluation of hip abductor muscles with use of magnetic resonance imaging. J Orthopaedic Res. 15: 888-893.

39. Mitomo S, Usa H, Hata M, Ogawa D, et al. (2018) Sequential changes in activity of hip abductors seen on muscle functional magnetic resonance imaging after hip abduction exercises. Bone Muscle 1: 1-4.

40. Jenner G, Foley JM, Cooper TG, Potchen EJ, et al. (1994) Changes in magnetic resonance images of muscle depend on exercise intensity and duration, not work. $J$ Appl Physiol 76: 2119-2124.

41. Meyer RA, Prior BM (2000) Functional magnetic resonance imaging of muscle. Exerc Sport Sci Rev 28: 89-92. [ crossref]

42. Neumann DA (2010) Kinesiology of the hip: a focus on muscular actions. J Orthop Sports Phys Ther 40: 82-94. [ crossref]

43. Sidorkewicz N, Edward DJ, Cambridge, McGill SM (2014) Examining the effects of altering hip orientation on gluteus medius and tensor fascae latae interplay during common non-weight-bearing hip rehabilitation exercises. Clin Biomech 29: 971-976.

44. Beck M, Sledge JB, Gautier E, Dora CF, Ganz R (2000) The anatomy and function of the gluteus minimus muscle. J Bone Joint Surg Br 82: 358-363. [ crossref] 
Mitomo S (2019) Sequential Changes in Activity of Hip Abductor Muscles after Side-Lying Hip Abduction Exercise with Different Directions Using Muscle Functional Magnetic Resonance Imaging

45. Al-Hayani A (2009) The functional anatomy of hip abductors. Folia Morphol (Warsz) 68: 98-103. [ crossref]

46. Semciw AI, Green RA, Pizzari T, Briggs C (2013) Verification of a standardized method for inserting intramuscular EMG electrodes into uniquely oriented segments of gluteus minimus and gluteus medius. Clin Anat 26: 244-252.

47. Pfirrmann CW, Chung CB, Theumann NH, Trudell DJ, et al. (2001) Greater trochanter of the hip: attachment of the abductor mechanism and a complex of three bursae - MR imaging and MR bursography in cadavers and MR imaging in asymptomatic volunteers. Radiology 221: 469-477.

48. O'Dwyer C, Sainsbury D, O'Sullivan K (2011) Gluteus medius muscle activation during isometric muscle contractions. J Sport Rehabil 20: 174-186.

49. Semciw AI, Pizzari T, Murley GS, Green RA (2013) Gluteus medius: an intramuscular EMG investigation of anterior, middle and posterior segments during gait. J Electromyogr Kinesiol 23: 858-864.
50. O'Sullivan K, Smith SM, Sainsbury D (2010) Electromyographic analysis of the three subdivisions of gluteus medius during weight-bearing exercises. Sports Med Arthros Rehabil Ther Technol 2: 1-9.

51. Selkowitz DM, Beneck GJ, Powers CM (2016) Comparison of Electromyographic Activity of the Superior and Inferior Portions of the Gluteus Maximus Muscle During Common Therapeutic Exercises. J Orthop Sports Phys Ther 46: 794-799. [ crossref]

52. Fisher MJ, Meyer RA, Adams GR, Foley JM, et al. (1990) Direct relationship between proton $\mathrm{T} 2$ and exercise intensity in skeletal muscle MR images. Invest Radiol 25: 480-485.

53. Ford KR, Nguyen AD, Dischiavi SL, Hegedus EJ, et al. (2015) An evidence-based review of hip-focused neuromuscular exercise interventions to address dynamic lower extremity valgus. Open Access J Sports Med 6: 291-303.

\section{Citation:}

Mitomo S, Usa H, Hata M, Ogawa D, Senoo A, Takei H (2019) Sequential Changes in Activity of Hip Abductor Muscles after Side-Lying Hip Abduction Exercise with Different Directions Using Muscle Functional Magnetic Resonance Imaging. Integr J Orthop Traumatol Volume 2 (3): 1-8. 